\title{
Polyhedra with few 3-cuts are hamiltonian
}

\author{
G. Brinkmann \\ Department of Applied Mathematics, Computer Science \& Statistics \\ Ghent University \\ 9000 Ghent, Belgium \\ Gunnar.Brinkmann@ugent. be \\ C. T. Zamfirescu* \\ Department of Applied Mathematics, Computer Science \& Statistics \\ Ghent University \\ 9000 Ghent, Belgium \\ and \\ Department of Mathematics \\ Babeş-Bolyai University \\ Cluj-Napoca, Roumania \\ czamfirescu@gmail.com
}

Submitted: Feb 16, 2018; Accepted: Apr 5, 2018; Published: Mar 8, 2019

(C) The authors. Released under the CC BY-ND license (International 4.0).

\begin{abstract}
In 1956, Tutte showed that every planar 4-connected graph is hamiltonian. In this article, we will generalize this result and prove that polyhedra with at most three 3-cuts are hamiltonian. In 2002 Jackson and Yu have shown this result for the subclass of triangulations. We also prove that polyhedra with at most four 3-cuts have a hamiltonian path. It is well known that for each $k \geqslant 6$ non-hamiltonian polyhedra with $k 3$-cuts exist. We give computational results on lower bounds on the order of a possible non-hamiltonian polyhedron for the remaining open cases of polyhedra with four or five 3-cuts.
\end{abstract}

Mathematics Subject Classifications: 05C45, 05C10, 05C40

\section{Introduction}

One of the classic results in graph theory is Whitney's theorem from 1931 that 4-connected triangulations of the plane are hamiltonian [9]. In the meantime several stronger versions

\footnotetext{
${ }^{*}$ Carol T. Zamfirescu is a PhD fellow at Ghent University on the BOF (Special Research Fund) scholarship 01DI1015
} 
of Whitney's theorem have been proven, the most prominent one the result of Tutte who showed in 1956 that all 4-connected planar graphs are hamiltonian [8]. Jackson and Yu proved that a hamiltonian cycle exists even if there are up to three 3-cuts in a triangulation [5] and in [3] it is proven that in 4-connected triangulations not only one hamiltonian cycle exists, but even a number linear in the number of vertices of the triangulation. Although also Tutte's theorem has been generalized in several ways - e.g. that in a 4-connected plane graph a hamiltonian cycle through any 2 edges exists [6] - the theorem by Jackson and $\mathrm{Yu}$ was not generalized to all 3-connected plane graphs with at most three 3-cuts. We will give this proof in this article.

In this article we will use the word polyhedron for 3-connected plane graphs and if $G=(V, E)$ for a set $V^{\prime} \subseteq V$ of vertices we write $G-V^{\prime}$ to denote the subgraph induced by the vertices in $V-V^{\prime}$ and for a set $E^{\prime}$ of edges we write $G-E^{\prime}$ to denote the graph $\left(V, E-E^{\prime}\right)$.

In Section 2 we give basic definitions and results that are tools used in the proofs in Section 3 where the main results are given and proven. The central theorem in that section is Theorem 16 stating that every polyhedron with at most three 3-cuts is hamiltonian. This theorem is also used to prove that every polyhedron with at most four 3-cuts is traceable. In Section 4 we prove that for the cases with four or five 3-cuts, where it is not yet known whether non-hamiltonian polyhedra exist, possible non-hamiltonian examples are 1-tough, so trivial examples as for six or more 3-cuts do not exist. We also prove the corresponding results for non-traceable polyhedra. Finally, in Section 5 we give the results of a computer search for non-hamiltonian polyhedra with four or five 3-cuts and non-traceable polyhedra with at most seven 3-cuts.

\section{Basic definitions and results}

Definition 1. - A vertex cutset in a graph $G=(V, E)$ is a set $S \subset V$ so that $G-S$ has more components than $G$.

A vertex cutset of size $k$ in a polyhedron $G$ is called trivial if it splits the graph into two components, one of which is a single vertex of degree $k$ in $G$.

- We call paths between two vertices $v, w$ vertex disjoint if they only share the vertices $v$ and $w$. If $X$ is a set of at least two vertices, we call paths starting at $v$ and ending in a vertex of $X$ vertex disjoint if they only share $v$.

- A polyhedron $G$ is called essentially 4-connected if all 3-cuts of $G$ are trivial. This implies that between two vertices of degree larger than 3 there are always at least 4 vertex disjoint paths.

Remark 2. An essentially 4-connected polyhedron $G$ with more than 6 vertices does not contain two vertices of degree 3 that share an edge of a triangle.

The (short) proof is left to the reader. 
Definition 3. Let $G$ be a polyhedron and $\{u, v, w\}$ a 3 -cut in $G$.

- If $\left(V^{\prime}, E^{\prime}\right)$ is a component of $G-\{u, v, w\}$, then the subgraph of $G$ induced by $V^{\prime} \cup\{u, v, w\}$ is called a closed component of $G-\{u, v, w\}$.

- If $\left(V^{\prime}, E^{\prime}\right)$ is a closed component of $G-\{u, v, w\}$, then $\left(V^{\prime}, E^{\prime} \cup\{\{u, v\},\{v, w\},\{w, u\}\}\right)$ is called an edge closed component of $G-\{u, v, w\}$.

Note that for a 3-cut $\{u, v, w\}$ in a polyhedron $G$ always exactly two closed components of $G-\{u, v, w\}$ exist. A third component would imply a plane embedding of a subdivision of $K_{3,3}$. The three vertices $u, v, w$ can not be contained in the boundary of the same face of $G$, as otherwise a vertex in the interior of the face can be connected to all three vertices - allowing a plane embedding of $K_{3,3}$.

Lemma 4. Let $G=(V, E)$ be a polyhedron with $k 3$-cuts and $\{u, v, w\}$ a 3-cut in $G$. Let $G^{\prime}=\left(V^{\prime}, E^{\prime}\right)$ be an edge closed component of $G-\{u, v, w\}$. Then we have:

- $G^{\prime}$ is planar and the vertices $u, v, w$ form a facial triangle in the (unique) embedding of $G^{\prime}$.

- Edge closed components of $G-\{u, v, w\}$ are polyhedra.

- For any two vertices in $G^{\prime}$ that are not both in $\{u, v, w\}$, there are at least as many vertex disjoint paths in $G^{\prime}$ as there are in $G$.

- Each 3-cut in $G^{\prime}$ is also a 3-cut in $G$.

- All edge closed components of $G-\{u, v, w\}$ together have at most $(k-1) 3$-cuts.

Proof. For $y \in V-V^{\prime}$ there are 3 vertex disjoint paths from $y$ to $\{u, v, w\}$ and as $\{u, v, w\}$ is a cut, none of these paths contains an element of $V^{\prime}-\{u, v, w\}$. The union of these paths is connected, so removing $V-V^{\prime}$ from $G$, the vertices $u, v, w$ are in the same face, so the edges $\{u, v\},\{v, w\},\{w, u\}$ can be added (if not yet present) inside the face where they form a facial triangle. So $G^{\prime}$ is planar. The fact that this embedding is unique will follow from the fact that $G^{\prime}$ is 3 -connected.

We will now prove that for any two vertices $a, b \in V^{\prime}, a \notin\{u, v, w\}$ and a path $P=v_{1}, \ldots, v_{n}$ from $a$ to $b$ in $G$, there is a path $T(P)$ from $a$ to $b$ in $G^{\prime}$ using only vertices from the set $\left\{v_{1}, \ldots, v_{n}\right\}$. We will also show that $T(P)$ uses at least 3 vertices unless $a=v_{1}$ and $v_{2}=b$. So, for a second path $P^{\prime}$ from $a$ to $b$ that is vertex disjoint with $P$, we have that $P \neq P^{\prime}$ implies $T(P) \neq T\left(P^{\prime}\right)$. This means that for any set $\left\{P_{1}, \ldots, P_{k}\right\}$ of vertex disjoint paths from $a$ to $b$ in $G$ the set $\left\{T\left(P_{1}\right), \ldots, T\left(P_{k}\right)\right\}$ has equal size and is also vertex disjoint.

Unless $v_{2}=b, v_{2}$ is also a vertex from $V^{\prime}$ different from $a, b$. If $P$ only contains vertices from $G^{\prime}$, we have found our path. Otherwise it contains a first edge $\left\{v_{i}, v_{i+1}\right\}$ not in $G^{\prime}$. W.l.o.g. we have $v_{i}=u$ and $v_{i+1} \notin V^{\prime}$. Then there is some maximal $j>i$ with $v_{j} \notin V^{\prime}$ and $v_{j+1} \in\{v, w\}$. Replacing $v_{i}, v_{i+1}, \ldots, v_{j}, v_{j+1}$ by $v_{i}, v_{j+1}$ we have a path in $G^{\prime}$ that 
uses only vertices from $\left\{v_{1}, \ldots, v_{n}\right\}$. So for $\{a, b\} \not \subset\{u, v, w\}$ there are at least 3 vertex disjoint paths from $a$ to $b$ as $G$ is a polyhedron.

For $\{a, b\} \subset\{u, v, w\}-$ w.l.o.g. $\{u, v, w\}=\{a, b, w\}-a, b$ and $a, w, b$ are two disjoint paths from $a$ to $b$ along the triangle. As for each vertex $z \notin\{u, v, w\}$ there are three vertex disjoint paths from $z$ to $\{a, b, w\}$, there is also at least one path from $a$ to $b$ not using $w$. So there are at least 3 vertex disjoint paths from $a$ to $b$.

These arguments imply that $G^{\prime}$ is 3 -connected, so the embedding is unique and $\{u, v\}$, $\{v, w\},\{w, u\}$ form a facial triangle in each embedding.

If $\left\{u^{\prime}, v^{\prime}, w^{\prime}\right\}$ is a 3 -cut in $G^{\prime}$ and $a, b$ are vertices in different components of $G^{\prime}-$ $\left\{u^{\prime}, v^{\prime}, w^{\prime}\right\}$, then at least one of them is not contained in $\{u, v, w\}$ and there is also no path from $a$ to $b$ in $G-\left\{u^{\prime}, v^{\prime}, w^{\prime}\right\}$, as there would be a corresponding path on the subset of vertices from $a$ to $b$ in $G^{\prime}-\left\{u^{\prime}, v^{\prime}, w^{\prime}\right\}$. This implies that $\left\{u^{\prime}, v^{\prime}, w^{\prime}\right\}$ is also a cut in $G$. As each 3-cut in an edge closed component is also a 3 -cut in $G$, but $\{u, v, w\}$ is a 3 -cut in $G$ but not in any of the edge closed components, this proves that there are at most $(k-1) 3$-cuts in all edge closed components together.

The number of all 3-cuts in the edge closed components together can be smaller than $k-1$. An easy example is a polyhedron with a vertex $v$ of degree 3 adjacent to 3 other vertices of degree 3 where the neighbourhoods of these 4 vertices are the only 3 -cuts. Choosing the neighbourhood of $v$ as a cut, we get one copy of $K_{4}$ and one 4-connected edge closed component, showing that the number of 3-cuts in the edge closed components can be smaller than $k-1$. On the other hand choosing another cut, the edge closed component has two 3-cuts left, showing that there is no unique decomposition tree like for triangulations (see [5]).

While in triangulations 3-cuts are separating triangles that lie properly inside each other, the relative position in general polyhedra can be more complicated. It is e.g. possible that vertices of a 3 -cut $\{u, v, w\}$ end up in different edge closed components of a 3 -cut $\left\{u^{\prime}, v^{\prime}, w^{\prime}\right\}$. This makes it worthwhile to explicitly state and prove the following lemma, which is trivial for triangulations:

Lemma 5. Each polyhedron $G$ with 3-cuts has a 3-cut $\{u, v, w\}$, so that at least one edge closed component $G^{\prime}$ of $G-\{u, v, w\}$ has no 3-cuts - that is: $G^{\prime}$ is 4-connected or isomorphic to $K_{4}$.

Proof. Let $\{u, v, w\}$ be a 3-cut, so that one of the edge closed components of $G-\{u, v, w\}$ has minimal cardinality among all edge closed components of 3-cuts of $G$. Let $G^{\prime}$ be this edge closed component and assume that $G^{\prime}$ contains a 3 -cut $\left\{u^{\prime}, v^{\prime}, w^{\prime}\right\}$. At least one of the vertices $\{u, v, w\}-$ w.l.o.g. $w$ - is not in $\left\{u^{\prime}, v^{\prime}, w^{\prime}\right\}$. Let $G^{\prime \prime}$ be the edge closed component of $G^{\prime}-\left\{u^{\prime}, v^{\prime}, w^{\prime}\right\}$ not containing $w$. We will prove that $G^{\prime \prime}$ is also an edge closed component of $G-\left\{u^{\prime}, v^{\prime}, w^{\prime}\right\}$ - contradicting the minimality of $G^{\prime}$.

Lemma 4 implies that $\left\{u^{\prime}, v^{\prime}, w^{\prime}\right\}$ is also a 3-cut in $G$. As $G^{\prime \prime}$ does not contain $w$, no edge containing $w$ is in $G^{\prime \prime}$ and as $u, v$ are neighbours of $w$ in $G^{\prime}$, they can only be in $G^{\prime \prime}$ if they are one of the vertices $u^{\prime}, v^{\prime}, w^{\prime}$. So edges between vertices of $\{u, v, w\}$ are only in $G^{\prime \prime}$ if they are between vertices of $\left\{u^{\prime}, v^{\prime}, w^{\prime}\right\}$, and therefore all edges in $G^{\prime \prime}-\left\{u^{\prime}, v^{\prime}, w^{\prime}\right\}$ are also 
in $G-\left\{u^{\prime}, v^{\prime}, w^{\prime}\right\}$. This implies that there is an edge closed component of $G-\left\{u^{\prime}, v^{\prime}, w^{\prime}\right\}$ containing $G^{\prime \prime}$ and due to the minimality of $G^{\prime}$ this component must properly contain $G^{\prime \prime}$. So there is a path $v_{1}, \ldots, v_{k}$ in $G-\left\{u^{\prime}, v^{\prime}, w^{\prime}\right\}$ from a vertex $v_{1}$ in $G^{\prime \prime}-\left\{u^{\prime}, v^{\prime}, w^{\prime}\right\}$ to a vertex $v_{k}$ not in $G^{\prime \prime}-\left\{u^{\prime}, v^{\prime}, w^{\prime}\right\}$, so that $v_{1}, \ldots, v_{k-1}$ are all in $G^{\prime \prime}-\left\{u^{\prime}, v^{\prime}, w^{\prime}\right\}$. In fact the vertex $v_{k}$ is also not in $G^{\prime}$, as otherwise we had a path proving that $v_{k} \in G^{\prime \prime}$. Note that $\left\{u^{\prime}, v^{\prime}, w^{\prime}\right\}$ is not a cutset in any of its edge closed components. As the edge $\left\{v_{k-1}, v_{k}\right\}$ is not in $G^{\prime}, v_{k}$ is not in $G^{\prime}$, so it must be in the other edge closed component of $G-\{u, v, w\}$, which is impossible as $v_{k} \notin\{u, v, w\}$. So we have a contradiction and $G^{\prime}$ does not contain a 3 -cut.

Lemma 6. If $G=(V, E)$ is a polyhedron and $\{u, v, w\}$ is a 3-cut in $G$, then in $G-\{\{u, v\}\}$ (which is equal to $G$ if $\{u, v\} \notin E$ ) the vertices $u$ and $v$ share a face

$$
u, u_{1}, \ldots, u_{k}, v, v_{1}, \ldots, v_{m}
$$

with $k \geqslant 1, m \geqslant 1$ and for all $1 \leqslant i \leqslant k, 1 \leqslant j \leqslant m$ the vertices $u_{i}$ and $v_{j}$ belong to different components of $G-\{u, v, w\}$.

Proof. Lemma 4 implies that in the two edge closed components the vertices $u, v, w$ form a facial triangle. Embedding the two edge closed components in a way that the facial triangle is once the outer face and once a bounded face, we can identify the two edge closed components along the edges of this triangle. This gives an embedding of a graph $G^{\prime}=\left(V, E^{\prime}\right)=(V, E \cup\{\{u, v\},\{v, w\},\{w, u\}\})$ containing $G$ and with the vertices of different components of $G-\{u, v, w\}$ on different sides of the triangle $(u, v, w)$. If one face containing the edge $\{u, v\}$ is $v, u, u_{1}, \ldots, u_{k}$ (so $k \geqslant 1$ as the smallest possible face size is a triangle) and the other is $u, v, v_{1}, \ldots, v_{m}$ (so $\left.m \geqslant 1\right)$, in $G^{\prime \prime}=\left(V, E^{\prime}-\{u, v\}\right.$ ) we get the face as described in the statement. As $(V, E \cup\{u, v\})$ is 3-connected, the embedding of $(V, E \cup\{u, v\})$ is unique, so equivalent to the subembedding of $G^{\prime}$ and $u, u_{1}, \ldots, u_{k}, v, v_{1}, \ldots, v_{m}$ is also a face in $G-\{\{u, v\}\}$. As $G$ is a subgraph of $G^{\prime}$, for $1 \leqslant$ $i \leqslant k, 1 \leqslant j \leqslant m$ the vertices $u_{i}$ and $v_{j}$ belong to different components of $G-\{u, v, w\}$.

Lemma 7. A polyhedron $G \neq K_{4}$ with $k 3$-cuts contains a spanning subgraph that can be obtained from a 4-connected polyhedron by deleting at most $k$ vertices.

Proof. We will prove this by induction in $k$. For $k=0$ the statement is trivial, so assume that $k>0$ and that $\{u, v, w\}$ is a 3 -cut in $G$. If $u, v, w$ do not form a separating triangle, assume w.l.o.g. that there is no edge $\{u, v\}$. We will construct a graph $G^{\prime}$ with at most $(k-1) 3$-cuts in which a vertex can be removed to obtain $G-\{\{u, v\}\}$. By induction a spanning subgraph of $G^{\prime}$ can be obtained by removing at most $k-1$ vertices from a 4-connected polyhedron, proving the result.

With the notation of Lemma 6 for the face of $G-\{\{u, v\}\}$ containing $u$ and $v$ we add a new vertex $z$ in the interior of the face $u, u_{1}, \ldots, u_{k}, v, v_{1}, \ldots, v_{m}$ and connect it to all vertices in the boundary to obtain a graph $G^{\prime}$. As $z$ connects vertices from the two different components of $G-\{u, v, w\}$, the set $\{u, v, w\}$ is not a cutset any more.

Assume now that there is a 3 -cut $\left\{u^{\prime}, v^{\prime}, w^{\prime}\right\}$ in $G^{\prime}$ that is not already a cutset in $G$. As the degree of $z$ is at least $4, z$ cannot form a trivial component and each component 
contains at least one vertex from $G$. Let $w_{1} \neq z, w_{2} \neq z$ be vertices from different components of $G^{\prime}-\left\{u^{\prime}, v^{\prime}, w^{\prime}\right\}$. So there is a path from $w_{1}$ to $w_{2}$ in $G-\left\{u^{\prime}, v^{\prime}, w^{\prime}\right\}$ which is not in $G^{\prime}-\left\{u^{\prime}, v^{\prime}, w^{\prime}\right\}$. As $\{u, v\}$ is the only edge that might be in $G-\left\{u^{\prime}, v^{\prime}, w^{\prime}\right\}$ but not in $G^{\prime}-\left\{u^{\prime}, v^{\prime}, w^{\prime}\right\}$ this edge $\{u, v\}$ is contained in this path and therefore $\{u, v\} \cap\left\{u^{\prime}, v^{\prime}, w^{\prime}\right\}=$ $\emptyset$. As $\{u, v\} \in E(G)$ due to the choice of $u, v$ the vertices $u, v, w$ form a separating triangle in $G$. We also have $z \in\left\{u^{\prime}, v^{\prime}, w^{\prime}\right\}$ as otherwise the edge $\{u, v\}$ could be replaced by the path $u, z, v$. For the same reason $u$ and $v$ belong to different components in $G^{\prime}-\left\{u^{\prime}, v^{\prime}, w^{\prime}\right\}$. This again implies that $w \in\left\{u^{\prime}, v^{\prime}, w^{\prime}\right\}$, so w.l.o.g. $\left\{u^{\prime}, v^{\prime}, w^{\prime}\right\}=\left\{u^{\prime}, z, w\right\}$ for some vertex $u^{\prime}$.

As $G-\{u, v, w\}$ has exactly two components, the interior and exterior of the separating triangle are connected and w.l.o.g. $u^{\prime}$ is in the exterior. So the interior is also connected in $G^{\prime}-\left\{u^{\prime}, z, w\right\}$. As $u, v$ both have neighbours in the interior, they belong to the same component - a contradiction.

So there is no 3 -cut $\left\{u^{\prime}, v^{\prime}, w^{\prime}\right\}$ in $G^{\prime}$ that is not already a cutset in $G$ and $G^{\prime}$ has at most $(k-1) 3$-cuts.

A graph $G=(V, E)$ is $k$-hamiltonian if for each set $S \subset V$ with $|S| \leqslant k$ the graph $G-S$ is hamiltonian. In [7] Thomas and Yu prove the following result which was originally conjectured by Plummer:

Theorem 8. 4-connected planar graphs are 2-hamiltonian.

Together with Lemma 7 this theorem implies immediately:

Theorem 9. - Polyhedra with at most two 3-cuts are hamiltonian.

- Polyhedra with at most one 3-cut are 1-hamiltonian.

Theorem 8 can obviously not be strengthened to imply 3-hamiltonicity, as removing three vertices from a 4-cut leads to a graph with a cutvertex, so in order to prove that polyhedra with at most three 3-cuts are hamiltonian, we need another strategy.

We use the following technical Lemma 11, which is proven in [5]. A circuit graph is a pair $(G, F)$ such that $G$ is a 2-connected plane graph and $F$ is a facial cycle of $G$ such that for any 2-cut $U$ of $G$, each component of $G-U$ contains a vertex of $F$. In a 3-connected graph, each facial cycle has this property. Let $X$ be a cycle in a circuit graph $(G, F)$. An $X$-bridge of $G$ is either a single edge of $G-E(X)$ with both ends on $X$, or a component $B$ of $G-V(X)$ together with the edges with one endpoint on $X$ and one in $B$ and the endpoints of these edges on $X$. We say that a cycle $X$ is an F-Tutte cycle if for any $X$-bridge $B$ we have that $|V(B) \cap V(X)| \leqslant 3$ and for any $X$-bridge $B$ containing an edge of $F$ we have that $|V(B) \cap V(X)| \leqslant 2$. Using this terminology, an $F$-Tutte cycle is a hamiltonian cycle if and only if all bridges are single edges. We call such bridges trivial.

We will use the following remark:

Remark 10. If $(G, F)$ is a circuit graph and $X$ is an $F$-Tutte cycle, then we have: 
- If $v \in F$ and $v \notin X$ then there are at most 2 vertex disjoint paths from $v$ to $X$. So there is a set $C$ of at most two vertices, so that $v$ and the elements of $X-C$ are in different components of $G-C$.

- If $v \notin F$ and $v \notin X$ then there are at most 3 vertex disjoint paths from $v$ to $X$. So there is a set $C$ of at most three vertices, so that $v$ and the elements of $X-C$ are in different components of $G-C$.

Proof. This is a direct consequence of the definition of an $X$-bridge.

\section{The main results about hamiltonian cycles and paths in poly- hedra with few 3-cuts}

In this section we will first prove some technical results. They will lead to the main theorem that polyhedra with at most three 3-cuts are hamiltonian, but can also be useful in other contexts.

Lemma 11 ([5], (3.2)). Let $(G, F)$ be a circuit graph, $r, z$ be vertices of $G$ and $e \in E(F)$. Then $G$ contains an $F$-Tutte cycle through $e, r$ and $z$.

A key to the main result in [5] is their Theorem 4.1. The following theorem is a corresponding result in the more general context of polyhedra. For $t=d=0$ it is also a direct consequence of Corollary 2 in [6].

Theorem 12. Let $0 \leqslant t, d \leqslant 2$ be integers with $t+d \leqslant 2$ and $G=(V, E)$ be an essentially 4-connected polyhedron.

Let $f$ be a face of $G$ containing a vertex $v$ with degree at least 4 with neighbouring edges $\{u, v\},\{v, w\}$ in the boundary and assume that except for $u, w$ - which can have any degree of at least 3 - there are exactly $t$ vertices $r_{1}, \ldots, r_{t}$ of degree 3 in $G$. Furthermore let $t_{1}=\left(u_{1}, v_{1}, w_{1}\right), \ldots, t_{d}=\left(u_{d}, v_{d}, w_{d}\right)$ be facial triangles different from $f$, so that in case $d=2$ not all three faces $f, t_{1}, t_{2}$ share a vertex of degree 3 (which would have to be $u$ or $w)$.

Then the following two equivalent statements are true:

- There is a hamiltonian cycle of $G$ containing $\{u, v\}$ and $\{v, w\}$ and $d$ edges $e_{1}, \ldots, e_{d}$ with $e_{j} \in t_{j}$ for $1 \leqslant j \leqslant d$ so that all edges $\{u, v\},\{v, w\}, e_{1}, \ldots, e_{d}$ are different.

- There is a path from $u$ to $w$ containing all vertices of $G$ but $v$, that contains $d$ distinct edges $e_{1}, \ldots, e_{d}$ with $e_{j} \in t_{j}$ for $1 \leqslant j \leqslant d$. Note that if $f$ is a triangle, the path contains no edge of $f$.

Proof. We will prove the first statement. The equivalence of the first and second statement follows immediately by adding, respectively removing the edges $\{u, v\}$ and $\{v, w\}$.

As $G$ is 3-connected, the edge $\{u, w\}$ is either not present in $G$ or the face $f$ is a triangle containing the edge $\{u, w\}$. Otherwise $\{u, w\}$ would be a 2 -cut. So we can assume that $f$ 
is a triangle $(u, v, w)$. If this was not the case, we could add the edge $\{u, w\}$ and remove it afterward as it will not be part of the hamiltonian cycle constructed.

Furthermore we can assume that $t_{1}, \ldots, t_{d}$ do not contain vertices of degree 3 : If a triangle contains a vertex of degree 3 , one of its edges will be contained in a hamiltonian cycle, so that we do not have to ensure this property. Note that this edge is only counted for one triangle, as for $d=2$, there are no vertices of degree 3 in $G$ (except maybe $u$ or $w$ which must not be shared). In case one triangle contains $u$ and $u$ has degree 3 (or analogously $w$ ), we also have to make sure that it contains another edge than $\{v, u\}$, but this is guaranteed as the edge $\{u, w\}$ is not contained in the hamiltonian cycle and the third edge is contained in both faces different from $f$.

It is sufficient to prove the theorem for $d=0$, as for $d>0$ we can add a new vertex of degree 3 in the interior of triangle $t_{i}, 1 \leqslant i \leqslant d$. As no vertex in the triangle has degree 3 , the result is an essentially 4-connected polyhedron with $d=0$ and $t$ increased by $d$. At the end we can modify the hamiltonian cycle through the new vertices by replacing the two edges incident with the new vertices by the edge connecting the endpoints of the edges on the triangle. The resulting hamiltonian cycle has the properties given in the theorem.

If $t=0$ we choose arbitrary vertices $r_{1}, r_{2} \notin\{u, v, w\}$ and if $t=1$ we choose an arbitrary vertex $r_{2} \notin\left\{u, v, w, r_{1}\right\}$.

Let $G^{\prime}$ denote the graph $G-\{v\}$. If $v$ is not contained in a 3-cut, $G^{\prime}$ is 3-connected and with $F^{\prime}$ the only face of $G^{\prime}$ that is not a face of $G$, the graph $\left(G^{\prime}, F^{\prime}\right)$ is an $F^{\prime}$-circuit graph.

If $v$ is contained in a 3 -cut, this 3 -cut is the neighbourhood of a vertex with degree 3 , as $G$ is essentially 4 -connected. So the 2-cuts of $G-\{v\}$ are exactly the neighbourhoods of former vertices of degree 3 that were adjacent to $v$. Together with $v$ each 2 -cut is a 3 -cut in $G$, so we have exactly 2 components, one component is trivial, and the cutvertices are on the boundary of $F^{\prime}$. The face $F^{\prime}$ contains at least 4 vertices and after the removal of a 2-cut each component contains vertices of $F^{\prime}$. So $\left(G^{\prime}, F^{\prime}\right)$ is an $F^{\prime}$-circuit graph.

Due to Lemma $11 G^{\prime}$ has an $F^{\prime}$-Tutte cycle $X$ through $\{u, w\}, r_{1}$ and $r_{2}$. We will show that $X$ is a hamiltonian cycle.

Assume first that there is a vertex $y \in F^{\prime}, y \notin X$. As $X$ is an $F^{\prime}$-Tutte cycle, this would imply that there is a 2-cut $C$ separating $y$ from the vertices in $\left\{u, w, r_{1}, r_{2}\right\}-C$. As there are at least two vertices in $\left\{u, w, r_{1}, r_{2}\right\}-C$ and as one component of each 2-cut is trivial - which can not be $y$ as otherwise $y$ would have degree 3 in $G$ and be on the cycle - at least one vertex of $\left\{u, w, r_{1}, r_{2}\right\}-C$ is in the same component as $y$. This is a contradiction, so all vertices of $F^{\prime}$ are contained in $X$.

Assume now that $y$ is not on $F^{\prime}$ and not on $X$. This implies that $\operatorname{deg}(y) \geqslant 4$ and that there are 4 vertex disjoint paths in $G$ from $y$ to $v$. All these paths must contain vertices of $F^{\prime} \subseteq X$, so the union of the first parts of these paths from $y$ to the first element from $X$ shows that the $X$-bridge containing $y$ has at least 4 endpoints on $X$ - a contradiction.

So $X$ is a hamiltonian cycle of $G^{\prime}$ and replacing the edge $\{u, w\}$ by the edges $\{u, v\}$ and $\{v, w\}$, we have a hamiltonian cycle of $G$ with the required properties.

Theorem 12 can be used to prove the following corollary: 
Corollary 13. Let $G$ be an essentially 4-connected polyhedron with at most one vertex of degree 3 and containing a facial triangle $(u, v, w)$ with degree of $v$ at least 4 . Then the following two equivalent statements are true:

- There is a hamiltonian cycle of $G$ containing $\{u, w\}$ as only edge of the triangle $(u, v, w)$. If there is no vertex with degree 3 and another triangle $(\bar{u}, \bar{v}, \bar{w})$ is given, the cycle can be chosen in a way that also a (different) edge of $(\bar{u}, \bar{v}, \bar{w})$ is contained.

- There is a hamiltonian path from u to $w$ containing no edge of the triangle $(u, v, w)$. If there is no vertex with degree 3 and another triangle $(\bar{u}, \bar{v}, \bar{w})$ is given, the path can be chosen in a way that also an edge of $(\bar{u}, \bar{v}, \bar{w})$ is contained.

Proof. As $v$ has degree at least 4 there are $\left\{u^{\prime}, v\right\},\left\{v, w^{\prime}\right\}$ that are both not contained in the triangle $(u, v, w)$, but another face $f$. Applying Theorem 12 for $d=1$ to the edges $\left\{u^{\prime}, v\right\},\left\{v, w^{\prime}\right\}$ and the triangle $t_{1}=(u, v, w)$, (resp. $d=2$ if $(\bar{u}, \bar{v}, \bar{w})$ is given) we get that there is a hamiltonian cycle through $\left\{u^{\prime}, v\right\},\left\{v, w^{\prime}\right\}$ that contains an edge of $(u, v, w)$ - but this edge can only be $\{u, w\}$. In case $(\bar{u}, \bar{v}, \bar{w})$ is given, the cycle also contains a (different) edge of $(\bar{u}, \bar{v}, \bar{w})$.

Lemma 14. Let $G$ be a polyhedron with at most one 3 -cut and let $(u, v, w)$ be a triangular face with $\operatorname{deg}(v) \geqslant 4$.

(i) Then there is a hamiltonian cycle of $G$ containing $\{u, v\}$ and $\{v, w\}$. This implies that there is a path from $u$ to $w$ containing no edges of the triangle $(u, v, w)$, but all vertices except $v$. If another triangle $(\bar{u}, \bar{v}, \bar{w})$ is given, the cycle (resp. path) can be chosen in a way that also a (different) edge of $(\bar{u}, \bar{v}, \bar{w})$ is contained.

(ii) There is also a hamiltonian cycle of $G$ containing $\{u, w\}$ as only edge of $(u, v, w)$. So there is a hamiltonian path from $u$ to $w$ containing no edges of $(u, v, w)$.

Proof. We will give the proof of (i) only for the stronger case where also $(\bar{u}, \bar{v}, \bar{w})$ is given.

Note that as $(u, v, w)$ and $(\bar{u}, \bar{v}, \bar{w})$ are triangular faces, none of them forms the unique 3 -cut and they are also faces in one of the edge closed components.

If the 3-cut is trivial, (i) and (ii) are direct consequences of Theorem 12 and Corollary 13 .

So assume that the 3 -cut $\left\{u^{\prime}, v^{\prime}, w^{\prime}\right\}$ is not trivial and let $G^{\prime}, G^{\prime \prime}$ be the two edge-closed components of $G-\left\{u^{\prime}, v^{\prime}, w^{\prime}\right\}$ sharing $\left\{u^{\prime}, v^{\prime}, w^{\prime}\right\}$. Then $G^{\prime}, G^{\prime \prime}$ are 4-connected and all vertices have degree at least 4. Assume that - w.l.o.g. - $u, v, w$ are in $G^{\prime}$.

(i) $G^{\prime}$ contains a hamiltonian cycle $H$ through $\{u, v\}$ and $\{v, w\}$ that contains also an edge - say $\left\{u^{\prime}, v^{\prime}\right\}$ - of the triangle $\left(u^{\prime}, v^{\prime}, w^{\prime}\right)$ different from $\{u, v\}$ and $\{v, w\}$ (Theorem 12) and a different edge in $(\bar{u}, \bar{v}, \bar{w})$ if that triangle is in $G^{\prime}$. If $\left\{u^{\prime}, v^{\prime}\right\}$ is the only edge of the triangle $\left(u^{\prime}, v^{\prime}, w^{\prime}\right)$ in $H$, we can apply Theorem 12 to $G^{\prime \prime}$ and get that $G^{\prime \prime}$ has a path from $u^{\prime}$ to $v^{\prime}$ containing all vertices but $w^{\prime}$. Replacing the edge $\left\{u^{\prime}, v^{\prime}\right\}$ with this path gives the hamiltonian cycle of $G$ with the required properties. If $(\bar{u}, \bar{v}, \bar{w})$ is in $G^{\prime \prime}$, the path can be chosen in a way that it contains an edge of $(\bar{u}, \bar{v}, \bar{w})$ not contained in $\left(u^{\prime}, v^{\prime}, w^{\prime}\right)$. 
If $H$ contains two edges of $\left(u^{\prime}, v^{\prime}, w^{\prime}\right)$ different from $\{u, v\}$ and $\{v, w\}-$ say $\left\{u^{\prime}, v^{\prime}\right\}$, $\left\{v^{\prime}, w^{\prime}\right\}$ - then we can apply Corollary 13 to conclude that $G^{\prime \prime}$ has a hamiltonian path from $u^{\prime}$ to $w^{\prime}$ using no edges of the triangle $\left(u^{\prime}, v^{\prime}, w^{\prime}\right)$ (but an edge of $(\bar{u}, \bar{v}, \bar{w})$ if that is in $\left.G^{\prime \prime}\right)$. Replacing the path $u^{\prime}, v^{\prime}, w^{\prime}$ in $H$ by this hamiltonian path gives the hamiltonian cycle of $G$ with the required properties.

(ii) Let $\{v, x\},\{v, y\}$ be edges in the same face of $G^{\prime}$ with $\{x, y\} \cap\{u, w\}=\emptyset$. Then there is a hamiltonian cycle $H$ through $\{v, x\}$ and $\{v, y\}$ that contains also an edge of the triangle $(u, v, w)$ (which must be $\{u, w\})$ and in case $\left\{u^{\prime}, v^{\prime}, w^{\prime}\right\} \neq\{v, x, y\}$ also a different edge in $\left(u^{\prime}, v^{\prime}, w^{\prime}\right)$ (Theorem 12). Precisely as in the first part, this hamiltonian cycle can be extended to a hamiltonian cycle of $G$ by applying Theorem 12, resp. Corollary 13. The extension in $G^{\prime \prime}$ does not use any edges that occur in $G^{\prime}$ (also no edges of the triangle $\left(u^{\prime}, v^{\prime}, w^{\prime}\right)$ which occur in both $-G^{\prime}$ and $\left.G^{\prime \prime}\right)$, so the resulting hamiltonian cycle contains $\{u, w\}$ as only edge from $(u, v, w)$.

Lemma 15. Let $G$ be a polyhedron with at most two 3-cuts and let $(u, v, w)$ be a triangular face. Then there is a hamiltonian cycle of $G$ containing an edge of $(u, v, w)$.

Proof. If all 3-cuts are trivial, this is a direct consequence of Theorem 12, so suppose that $\left\{u^{\prime}, v^{\prime}, w^{\prime}\right\}$ is a nontrivial 3-cut, that $G^{\prime}, G^{\prime \prime}$ are the two edge closed components and that $G^{\prime}$ contains the face $(u, v, w)$.

As $G^{\prime}$ and $G^{\prime \prime}$ together have at most one 3-cut, at most one of $u^{\prime}, v^{\prime}, w^{\prime}$ has degree 3 in one of them, so assume that $\operatorname{deg}_{G^{\prime}}\left(v^{\prime}\right) \geqslant 4$ and $\operatorname{deg}_{G^{\prime \prime}}\left(v^{\prime}\right) \geqslant 4$. Lemma 14 (i) implies that there is a path from $u^{\prime}$ to $w^{\prime}$ in $G^{\prime}$ containing all vertices but $v^{\prime}$ that contains also an edge of $(u, v, w)$. In $G^{\prime \prime}$ we can apply Lemma 14 (ii) to conclude that $G^{\prime \prime}$ contains a hamiltonian path from $u^{\prime}$ to $w^{\prime}$ using only edges of $G^{\prime \prime}$ not contained in the triangle $\left(u^{\prime}, v^{\prime}, w^{\prime}\right)$.

The union of these paths gives the hamiltonian cycle with the described properties.

Theorem 16. Every polyhedron $G$ with at most three 3-cuts is hamiltonian.

Proof. If all 3-cuts are trivial, this is a direct consequence of Theorem 12 with $t=2$ and choosing one of the vertices with degree 3 as $u$.

So assume that there is a nontrivial cut $\{u, v, w\}$. Splitting $G$ at this cut we get two edge closed components $G^{\prime}$ and $G^{\prime \prime}$ with together at most two 3-cuts.

If one of them - w.l.o.g. $G^{\prime \prime}$ - has no 3-cuts, then $G^{\prime}$ has at most two 3-cuts and we can apply Lemma 15 . We conclude that $G^{\prime}$ has a hamiltonian cycle containing an edge of $(u, v, w)$. Assume that this edge is $\{u, w\}$. As $G^{\prime \prime}$ does not have 3 -cuts, we can replace $\{u, w\}$ by a path from $u$ to $w$ in $G^{\prime \prime}$ containing all vertices but $v$ (Theorem 12).

So assume that $G^{\prime}$ and $G^{\prime \prime}$ both have one 3-cut. This implies that in each of $G^{\prime}, G^{\prime \prime}$ at most one of $u, v, w$ can have degree 3. So one vertex - w.l.o.g. $v$ has degree 4 in both edge-closed components. Then Lemma 14 implies that $G^{\prime}$ has a path from $u$ to $w$ containing no edges of $(u, v, w)$ and all vertices but $v$ and that $G^{\prime \prime}$ has a hamiltonian path from $u$ to $w$ containing no edges of $(u, v, w)$. Combining these paths gives a hamiltonian cycle of $G$. 
Corollary 17. If all polyhedra with at most $k 3$-cuts are hamiltonian, then all polyhedra with at most $(k+1) 3$-cuts are traceable (that is: contain a hamiltonian path).

Especially: all polyhedra with at most four 3-cuts are traceable.

Proof. Let $G$ be a polyhedron with at most $(k+1) 3$-cuts.

Theorem 12 implies that for any two vertices $u, v$ in a triangle $(u, v, w)$ of a 4 -connected polyhedron, there is a path from $u$ to $v$ through all vertices but $w$. The same is obviously also true for $K_{4}$.

Let $\{u, v, w\}$ be a 3 -cut in $G$ so that one of the edge closed components, say $G^{\prime}$, has no 3 -cut. The other edge closed component $G^{\prime \prime}$ has at most $k 3$-cuts, so there is a hamiltonian cycle $H$ in $G^{\prime \prime}$. There are 3 possibilities how this hamiltonian cycle passes through edges of the triangle $(u, v, w)$ :

- $H$ uses no edge of $u, v, w$ :

So w.l.o.g. $H=u, u_{1}, \ldots, v, v_{1}, \ldots, w, w_{1}, \ldots, u$. Combining $H-\left\{\left\{u, u_{1}\right\},\left\{v, v_{1}\right\}\right\}$ with a path in $G^{\prime}$ from $u$ to $v$ through all vertices but $w$ gives a hamiltonian path in $G$ from $u_{1}$ to $v_{1}$.

- $H$ uses only the edge $\{u, v\}$ of $u, v, w$ :

Combining $H-\{\{u, v\}\}$ with a path in $G^{\prime}$ from $u$ to $v$ through all vertices but $w$ even gives a hamiltonian cycle in $G$.

- $H$ uses the edges $\{u, v\},\{v, w\}$ of $u, v, w$ :

Combining $H-\{\{u, v\},\{v, w\}\}$ with a path in $G^{\prime}$ from $u$ to $v$ through all vertices but $w$ gives a hamiltonian path in $G$ from $v$ to $w$.

On the other hand one can prove:

Lemma 18. For each $d \geqslant 8$ there exist non-traceable triangulations with $d$ 3-cuts.

Proof. For each $n \geqslant 6$ there is a double wheel that provides an example of a 4 -connected triangulation with $n$ vertices and $2 n-4$ triangular faces.

For even $d \geqslant 8$ take a double wheel (or any other 4-connected triangulation) $T$ with $d$ triangular faces. $T$ has $n=\frac{d}{2}+2$ vertices. As $d \geqslant 8$ we have $d-n \geqslant 2$. If we insert single vertices into $d$ triangular faces of $T$ and connect them to the vertices in the surrounding triangle, we obtain a triangulation $T^{\prime}$, that has exactly $d$ 3-cuts: each triangle into which a vertex was inserted is a 3 -cut and for each other set $C$ of 3 vertices, the subgraph $T$ is still connected and each new vertex that is not in $C$ is connected to at least one remaining vertex in $T-C$ - so $T^{\prime}-C$ is connected. This proves that the $d$ triangles form the only 3 -cuts.

Removing the $n$ vertices of $T$, we have $d \geqslant n+2$ components left, which proves that there can not be a hamiltonian path as it would have to pass all $d$ components with less than $d-1$ intermediate points from $T$, which is obviously impossible.

Denoting the number of components of a graph as $c(G)$, Hendry [4] defined the scattering number $s(G)$ as $s(G)=\max \{c(G-X)-|X| \quad \mid \quad X \subset V(G), c(G-X) \geqslant 2\}$. 
The scattering number can be considered as a generalization of the concept of toughness. So in the language of Hendry [4] we have proven that $s\left(T^{\prime}\right) \geqslant 2$ and illustrated the well known fact that it has to be at most 1 to be traceable. Graphs with scattering number at least 2 can be considered trivially non-traceable.

\section{The toughness and scattering number of polyhedra with few 3-cuts}

As for 6 or more 3-cuts there are non-hamiltonian polyhedra even in the subclass of triangulations [2], the only remaining cases for which it is not decided whether nonhamiltonian polyhedra with these numbers of cuts exist are four or five 3-cuts. For traceability the undecided cases are five, six or seven 3-cuts. In this section we will prove that there are no trivially non-hamiltonian (not 1-tough) polyhedra with four or five 3cuts and no trivially non-traceable (scattering number at least two) polyhedra with five, six or seven 3 -cuts.

In [2] the authors prove that triangulations with four or five 3-cuts are necessarily 1-tough, which means that no easy counterexamples exist. This result is based on the following lemma.

A graph $G=(V, E)$ is 1-tough if $s(G) \leqslant 0$, so for all cutsets $S \subset V$ of $G$ we have that $c(G-S) \leqslant|S|$. Note that in a triangulation a 3-cut consists of vertices of a separating triangle and the vertices of each separating triangle form a 3-cut.

Lemma 19. [2]

In a triangulation $T=(V, E)$ with at most $d$ separating triangles we have for each cutset $S \subset V: c(T-S) \leqslant|S|-2+\left\lfloor\frac{d}{2}\right\rfloor$.

So for $d \leqslant 5$ we have $c(T-S) \leqslant|S|$ which implies that triangulations with at most 5 separating triangles are 1-tough.

For $d \leqslant 7$ we have $c(T-S) \leqslant|S|+1$ which implies that for triangulations with at most seven 3 -cuts the scattering number is at most 1.

One may hope that as polyhedra can have much less edges than triangulations, the situation is different and that there might be polyhedra with four or five 3-cuts that are not 1-tough or polyhedra with five, six or seven 3-cuts and scattering number at least 2 . We will now prove that this is not the case.

Remark 20. For each polyhedron $G=(V, E)$ with $d$ 3-cuts and each cutset $S \subset V$, there is a triangulation $T_{S, G}=\left(V, E^{\prime}\right)$ containing $G$ as a subgraph with at most $d$ 3-cuts, so that $c\left(T_{S, G}-S\right)=c(G-S)$.

Proof. First note that for two non-adjacent vertices $v, v^{\prime}$ of a face $f$ of $G$, we have that $\left\{v, v^{\prime}\right\} \notin E$, as otherwise $\left\{v, v^{\prime}\right\}$ would be a 2-cut. This implies that we can add the edge $\left\{v, v^{\prime}\right\}$ inside $f$ without producing double edges. Adding an edge, no new cuts can occur, so that the number of 3-cuts can not increase.

Let $A_{1}, \ldots, A_{k}$ be the components of $G-S$. We call a set of two vertices $v, v^{\prime} \in V$ connecting if $v$ and $v^{\prime}$ are in different components of $G-S$ and non-connecting otherwise. 
Obviously all edges of $G$ are non-connecting. For any graph $G^{\prime}=\left(V, E^{\prime}\right)$ containing $G$ that has only non-connecting edges we have that $c\left(G^{\prime}-S\right)=c(G-S)$.

Let now $T=\left(V, E^{\prime}\right)$ be a polyhedron that has only non-connecting edges, contains $G$ and has the maximum number of edges among all polyhedra with these properties.

Assume that $T$ has a face $f$ that is not a triangle. Then $f$ does not contain vertices from $S$, as a vertex $v \in S$ can be connected to any other vertex in $f$-except to its two neighbours - by a non-connecting edge. This is in contradiction to the maximality of $T$. The same would be true if $f$ contained only vertices from the same component of $G-S$. So $f$ contains vertices from different components of $G-S$. As there are no edges in $G$ or $T$ between different components of $G-S$, they must be separated by vertices of $S$. This contradicts the fact that no vertices of $S$ are in $f$.

So all faces are triangles and $T$ is a triangulation with the desired properties.

Note that the triangulation $T_{S, G}$ is in general not uniquely determined.

Corollary 21. In a polyhedron $G=(V, E)$ with at most $d$ 3-cuts we have for each cutset $S \subset V: c(G-S) \leqslant|S|-2+\left\lfloor\frac{d}{2}\right\rfloor$.

So for $d \leqslant 5$ we have $c(G-S) \leqslant|S|$ which implies that polyhedra with at most five 3 -cuts are 1 -tough. For $d \leqslant 7$ we have $c(T-S) \leqslant|S|+1$ which implies that for polyhedra with at most seven 3 -cuts the scattering number is at most 1 .

Proof. With Remark 20 and Lemma 19 this follows directly, as for each set $S$ we have $c(G-S) \leqslant c\left(T_{S, G}-S\right) \leqslant|S|-2+\left\lfloor\frac{d}{2}\right\rfloor$.

\section{Computational results}

The case of four or five 3-cuts is even in the subclass of triangulations, where one has more information on the polyhedron, still undecided. One would expect that - in case polyhedra resp. triangulations with four or five 3-cuts are all hamiltonian - the proof for triangulations is easier and found first.

If on the other hand there are polyhedra with four or five 3-cuts that are nonhamiltonian, it is possible that they only exist outside the class of triangulations or one might expect the size of a smallest counterexample outside the class of triangulations to be smaller than the size of the smallest triangulation that is a counterexample, as removing edges from triangulations (without increasing the number of 3-cuts) might destroy hamiltonian cycles. To this end or to get at least a lower bound on the size of a possible non-hamiltonian polyhedron with four or five 3-cuts, we implemented a computer search. This computer search is based on the program plantri [1] to which we added a filter that allows to restrict the generation to polyhedra with a number of 3-cuts inside a given range (in our case 4 or 5 ). The program generates more than 500, 000 non-isomorphic polyhedra - each with four or five 3-cuts - per second on an Intel Quad CPU Q8200 running at $2.33 \mathrm{GHz}$.

In plantri polyhedra are generated by successively removing edges from triangulations but keeping the graph 3-connected. This implies that the number of 3-cuts can never 
decrease when removing edges and no further edges have to be removed in the recursive process when the number of 3-cuts exceeds the upper limit. Polyhedra are output only if the number of 3-cuts lies within the range given by the upper and lower limit, but polyhedra with fewer 3-cuts than the lower limit also occur inside the generation process.

We count 3-cuts by using the following easy lemma:

Lemma 22. Three vertices $u, v, w$ form a 3 -cut in a polyhedron $G=(V, E)$ if and only if any two of them share a face, but not all three of them share a face.

Proof. Assume first that $\{u, v, w\}$ is a 3 -cut. If all three shared a face, this would make a plane embedding of $K_{3,3}$ possible, so they do not share a face. On the other hand the two edge closed components can be identified along the triangles $(u, v, w)$ to give an embedding of $(V, E \cup\{\{u, v\},\{v, w\},\{w, u\}\})$, which is unique and in which any two of them share a face. So any two of them also share a face in $G$, which can be obtained by possibly deleting edges.

Assume now that each two of them, but not all three share a face. Then we can connect each pair of them (if not yet connected) by an edge inside the corresponding face and obtain a triangle $(u, v, w)$. If this was a facial triangle, then all three of them would share a face, so it is a non-facial triangle separating the inside from the outside and $u, v, w$ form a 3-cut.

For each triangulation we start with, we store for each vertex $v$ the set of vertices that $v$ shares a face with. This set is stored as a bit set. Furthermore we store each set of 3 vertices that share a face - that is: all sets of facial triangles. Then the condition from Lemma 22 is tested for all triples $u<v<w$ of vertices and the 3-cuts are counted.

For each number $i>0$ of edges removed from a triangulation the vector of bit sets coding for each vertex the set of vertices it shares a face with is updated from the corresponding vector for $i-1$. The only vertices for which the sets change are vertices in the new face that is the union of the two faces $f_{1}, f_{2}$ on two sides of the edge just removed. Possible new 3 -cuts are only 3 -cuts that contain one vertex $u$ from $f_{1}$ (but not $f_{2}$ ), one vertex $w$ from $f_{2}$ (but not $f_{1}$ ) and one vertex neither in $f_{1}$, nor in $f_{2}$. If the vector storing the bit sets of vertices is bs [] - so bs [u] is the set of all vertices that $u$ shares a face with - and newface is a bit set with all vertices in the new face, then for each $u, v$ as above counting the 3-cuts they are involved in comes down to counting the 1-bits in bs $[u]$ \& bs [v] \& newface which is a very efficient operation. Note that all these 3 -cuts are new in the sense that they were no 3-cuts before the last edge was removed. If they had been 3-cuts before, after removal the graph had at least 3 components and would either allow a plane embedding of $K_{3,3}$ or have a 2-cut.

When generating only polyhedra with four or five 3-cuts, the time needed for determining the number of 3-cuts is about $5 \%$ of the generation time. We tested the implementation by comparing the results to those of a very simple filter that filtered all polyhedra by removing all sets of three vertices and testing the rest for being connected. The results were compared for all polyhedra on up to 15 vertices (more than 25, 000, 000, 000 graphs) by once counting 3 -cuts with the simple program and once generating only those with a given number of cuts. There was complete agreement. 
To check hamiltonicity, we added a filter to plantri. The filter uses a simple branch and bound approach trying to build a hamiltonian cycle by successively increasing a path. There are only two look-aheads used. They are also implemented by very efficient bit operations:

- If not all vertices have been visited and the start vertex of the path has no neighbour in the set of unvisited vertices, the routine backtracks.

- If one of the still unvisited vertices has less than two neighbours in the set consisting of the still unvisited vertices and the start and end vertex of the path, the routine backtracks.

The routine takes advantage of the way that plantri constructs polyhedra: for triangulations a hamiltonian cycle is searched and stored in case one is found. As all other polyhedra are constructed by removing an edge from the ancestor in the generation, a previously constructed hamiltonian cycle may still be present. So for polyhedra that are not triangulations and with a hamiltonian ancestor, it is first tested whether the last edge removed belonged to the hamiltonian cycle stored for the ancestor of the polyhedron. Only if this is not the case, a new hamiltonian cycle is constructed and stored. The profile for up to 15 vertices showed that hamiltonicity testing took less than $20 \%$ of the total time, but the ratio increased slowly with the number of vertices. The hamiltonicity testing routine was checked by comparing the number of hamiltonian polyhedra on up to 15 vertices to numbers obtained by an extremely simple independent program. There was complete agreement.

Analogous to the case of four or five 3-cuts where the existence of hamiltonian cycles is still open, the existence of hamiltonian paths for five, six or seven 3-cuts is still open.

The filter checking traceability is very similar to the one checking hamiltonicity, except that it starts at every vertex and uses slightly modified bounding criteria. With the set $S$ consisting of the still unvisited vertices and the end vertex of the path (but not the start vertex), the only bounding criteria used are:

- If one of the still unvisited vertices has no neighbour in $S$ the routine backtracks.

- If at least two of the still unvisited vertices have less than two neighbours in $S$, then the routine backtracks.

The profile for up to 14 vertices showed that traceability testing took about $22 \%$ of the total time, but also here the ratio increased slowly with the number of vertices. The traceability testing routine was checked by comparing the number of non-traceable polyhedra on up to 15 vertices to numbers obtained by an extremely simple independent program. There was complete agreement. The first non-traceable polyhedra occur for 14 vertices.

The result of the computations performed on a cluster of the HPC infrastructure at Ghent University are: 
Lemma 23. - There are no non-hamiltonian polyhedra with at most five 3-cuts on up to 19 vertices.

- There are no non-traceable polyhedra with at most seven 3-cuts on up to 18 vertices.

The total CPU time for the search for non-hamiltonian polyhedra was approximately 3 years. During the test for 19 vertices alone 45, 849, 541,741, 643 polyhedra were tested for their number of cuts and the existence of a hamiltonian cycle. The search for non-traceable polyhedra took about 250 days and 12,229, 809,370,343 polyhedra on 18 vertices were tested for their number of cuts and the existence of hamiltonian paths.

\section{Acknowledgements}

The computational resources (Stevin Supercomputer Infrastructure) used to obtain the extensive computational results were provided by Ghent University, the Hercules Foundation and the Flemish Government - department EWI. We want to thank Brendan McKay for helpful discussions on this topic.

\section{References}

[1] G. Brinkmann and B.D. McKay. Fast generation of planar graphs. MATCH Commun. Math. Comput. Chem., 58(2):323-357, 2007. See http://cs.anu.edu.au/ $\sim \mathrm{bdm} /$ index. html.

[2] G. Brinkmann, J. Souffriau, and N. Van Cleemput. On the strongest form of a theorem of Whitney for hamiltonian cycles in plane triangulations. J. Graph Theory, 83(1):78-91, 2016.

[3] G. Brinkmann, J. Souffriau, and N. Van Cleemput. On the number of hamiltonian cycles in triangulations with few separating triangles. J. Graph Theory, 87(2):164$175,2018$.

[4] G.R.T. Hendry. Scattering number and extremal non-hamiltonian graphs. Discrete Math., 71:165-175, 1988.

[5] B. Jackson and X. Yu. Hamilton cycles in plane triangulations. J. Graph Theory, 41(2):138-150, 2002.

[6] D.P. Sanders. On paths in planar graphs. J. Graph Theory, 24(4):341-345, 1997.

[7] R. Thomas and X. Yu. 4-connected projective-planar graphs are hamiltonian. $J$. Comb. Theory B, 1:114-132, 1994.

[8] W.T. Tutte. A theorem on planar graphs. Trans. Am. Math. Soc., 82:99-116, 1956.

[9] H . Whitney. A theorem on graphs. Ann. Math., 32(2):pp. 378 - 390, 1931. 